\section{PA-120 THE UTILITY OF FINGERPRINT-BASED PARTICIPANT IDENTIFICATION AND CONSENTING IN CLINICAL TRIALS IN DEVELOPING COUNTRY SETTINGS}

Joseph Okebe, Pa Modou Cham, Ismaela Abubakar, Bai Lamin Dondeh, Umberto D'Alessandro. MRC, The Gambia

\subsection{6/bmjgh-2016-000260.149}

Background Involvement in clinical research requires evidence of informed participant consent, and in many low-income countries with high illiteracy rates, this is done by a fingerprint impression on paper. Due to poor quality of the impression, the individual is often untraceable and this reduces the quality of the process. The study assesses the potential usefulness of fingerprints for consenting and automated participant identification.

Methods As part of a survey in villages in the North Bank West region of The Gambia, individuals of all ages were invited to provide a fingerprint scan to update the pre-trial census records. Using commercial software, scanned impressions were stored on encrypted templates and linked to a unique identifier. A scan is successful if any of the five fingers on the left hand is captured but documented as a failure if none of the five fingers on the left hand records an impression on the scanner. We determined the proportion of successful attempts, and the effect of age and gender on the successful scan using a logistic regression model.

Results A total of 5204 persons were scanned with $74.7 \%$ successes for any finger; $70.3 \%(1550 / 2206)$ in males and $78.0 \%$ $(2339 / 2998)$ in females and gender was strongly associated with success rate $($ Chi $2<0.001)$. The success rate in children $<5$ years was $70.6 \%(726 / 1029)$ but lowest in adult males $\geq 40$ years; $29.7 \%$ (96/323). The odds of a successful scan were lower in males (adjusted OR 0.53; 95\% CI: 0.46-0.61; p $>0.001$ ) and highest between ages $5-25$ years (OR 8.32; 95\% CI: 6.96-9.95; $\mathrm{p}<0.001)$ compared to adults $\geq 40$ years.

Conclusions The use of fingerprint-based identification is promising. However, recognition rates are lowest in adult males, perhaps due to occupational practice. Potential for improving sensitivity and application in data retrieval and documenting consent is being explored. 